\title{
Electroosmotic Flow of a General Electrolyte Solution through a Fibrous Medium
}

\author{
Eric Lee, Yen-Shern Lee, Fong-Yuh Yen, and Jyh-Ping Hsu ${ }^{1}$ \\ Department of Chemical Engineering, National Taiwan, University, Taipei, Taiwan 10617, ROC
}

Received June 4, 1999; accepted November 9, 1999

\begin{abstract}
Theelectroosmotic flow of a general electrolytesolution through a fibrous medium is modeled theoretically taking the effect of doublelayer polarization into account. The result obtained is applicable to an arbitrary level of electrical potential. Weshow that if the effect of double-layer polarization is neglected using the linearized PoissonB oltzmann equation will underestimateelectroosmotic velocity. The deviation becomes inappreciable, however, if $\kappa a$ is either very large or very small, $\kappa$ and $a$ being, respectively, the reciprocal Debye length and the radius of a fiber. If the surface potential is high, the variation of electroosmotic velocity as a function of $\kappa a$ may exhibit a local maximum and a local minimum, and the larger the porosity of the fibrous medium the lower the level of surface potential for the local extremals to occur. If $\kappa a$ is small, the effect of surface potential on the electroosmotic velocity is more significant than that of double-layer polarization, and the reverse is true if $\kappa a$ is large. ${ }^{2} 2000$ Academic Press

Key Words: electroosmotic flow; electrolyte solution; fibrous medium; electrokinetic equations; double-layer polarization.
\end{abstract}

\section{INTRODUCTION}

Electroosmosis, the flow of liquid induced by an applied electric field, is one of the basic electrokinetic phenomena that finds many applications in practice. The flow of fluid occurs when an electric field is applied across a porous medium having a charged surface. The application of the electric field causes the ions in the double layer near the charged surface to migrate toward either cathode or anode. Since the ions are predominantly of one sign, their motion gives rise to a body force, which exerts on the liquid in the double layer, and the flow of liquid occurs.

Levine and Neale (1-3) analyzed both the electrophoresis and the electroosmosis of a swarm of spheres under the condition of low electrical potential. Their results are applicable for double layers of arbitrary thickness. They showed that the overlapping of adjacent double layers leads to a drastic decrease in both electrophoretic mobility and electroosmosis velocity. Kozak and Davis $(4,5)$ extended the analysis of Levine and Neale for electrophoresis to all levels of electrical potential taking the effect of double-layer polarization into account. The interaction between adjacent double layers, however, is neglected. The result

${ }^{1}$ To whom correspondence should be addressed. obtained in their analysis, therefore, is appropriate for thin double layers only. Ohshima (6) proposed an approximate method in which the tedious integration in the evaluation of electrophoretic mobility in Levine and Neale (3) can be avoided, and a simple analytic formula was derived. The deviation of the approximate analytical expression from the original one is less than $4 \%$. The analysis of Levine and Neale was also extended by Lee et al. (7) in a recent study to an arbitrary thickness of double layer and all levels of surface potential taking the effect of double-layer polarization into account. Their results reveal that both surface potential and double-layer polarization have a significant effect on the electrophoretic behavior of a concentrated colloidal dispersion. Kozak and Davis (8) applied the theory of Levine and Neale (3) to examine the electroosmotic phenomenon in a fibrous, porous medium under the condition that the effect of double-layer polarization is negligible. The medium is simulated by an array of circular cylinders, and the flow of the liquid phase to the axes of the cylinders is normal. Their results are applicable to an arbitrary thickness of double layer, but are limited to low electrical potential. Ohshima (9) proposed an approximate method similar to that of Ohshima (6) to arrive at a simple analytic electroosmotic velocity for flow through a fibrous, porous medium at low electrical potential. The deviation of the approximate result from that of Kozak and Davis is less than $6 \%$.

In this study, the analysis of Levine and Neale (3) is extended to an arbitrary level of potential and double-layer thickness; the effect of double-layer polarization is taken into account also. The general electrokinetic equations, which include the PoissonBoltzmann equation for electrical field and the Navier-Stokes equation for flow field, are solved simultaneously by employing the pseudospectral method based upon Chebyshev polynomials proposed in our previous studies for the case of a sphere in a spherical cavity $(10,11)$ and spheres in a concentrated suspension (7).

\section{THE ORY}

Let us consider a liquid containing $z_{1}: z_{2}$ electrolyte flow through an array of parallel, charged cylinders of radius $a, z_{1}$ and $z_{2}$ being, respectively, the valences of cations and anions. Referring to Fig. 1, the flow of liquid to the axes of the cylinders is normal and is parallel to an applied electrical field $\vec{E}$. The 


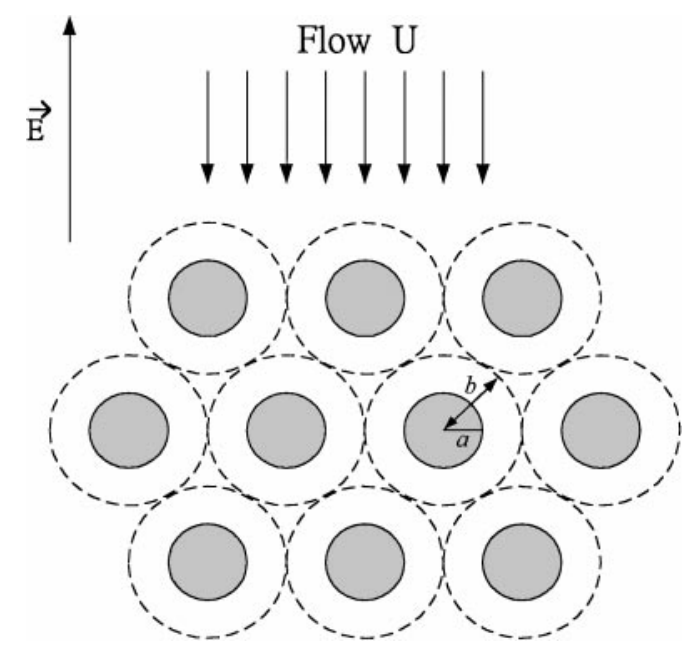

FIG. 1. Schematic representation of the system under consideration. The fibrous medium is simulated by parallel cylinders of radius $a$. The flow of liquid to the axes of the cylinders is normal, and the electric field $\vec{E}$ is in the direction of flow.

unit cell model proposed by Kuwabara (12) is adopted where the system under consideration is simulated by a representative cylinder of radius $a$ surrounded by a fluid envelope of radius $b$. The porosity of the fibrous medium, $H$, is obtained by

$$
H=1-(a / b)^{2} \text {. }
$$

The cylindrical coordinates $(r, \theta, z)$, with their origin located at the center of the representative cylinder, are adopted in the discussion below. Suppose that the liquid phase is incompressible and has constant physical properties, then the electroosmotic behavior of the system can be described by the set of electrokinetic equations describing the electric and flow fields.

\section{Electrical Potential}

Suppose that the time scale for double-layer relaxation is much smaller than that of the motion of fluid, then the spatial variation of the electrical potential $\phi$ can be described by the Poisson-Boltzmann equation

$$
\nabla^{2} \phi=-\frac{\rho}{\varepsilon},
$$

with

$$
\rho=\sum_{i} n_{i} z_{i} e
$$

where $\nabla$ represents the gradient operator, $\varepsilon$ and $\rho$ are, respectively, the permittivity of the liquid phase and the space charge density.

\section{Conservation of Ions}

At steady state, the conservation of ions leads to

$$
\nabla \cdot\left\{D_{j}\left[\nabla n_{j}+\frac{z_{j} e n_{j}}{k T} \nabla \phi\right]-n_{j} \vec{v}\right\}=0
$$

where $D_{j}, n_{j}$, and $z_{j}$ are the diffusivity, the number concentration, and the valence of ion species $j ; e$ and $\phi$ are, respectively, the elementary charge and the electrical potential; and $k$ and $T$ are the Boltzmann constant and the absolute temperature, respectively.

\section{Flow Field}

The flow field is described by the Navier-Stokes equation. Assuming creeping flow, we have

$$
\begin{aligned}
\mu \nabla^{2} \vec{v}-\nabla p-\rho \nabla \phi & =0 \\
\nabla \cdot \vec{v} & =0,
\end{aligned}
$$

where $p$ and $\mu$ are, respectively, the pressure and the viscosity of the liquid phase.

Suppose that $\phi$ can be decomposed as (13)

$$
\phi=\phi_{1}+\phi_{2}
$$

where $\phi_{1}$ and $\phi_{2}$ represent, respectively, the electrical potential that would exist in the absence of an electrical field and that due to the imposed electrical field. According to O'Brien and White (13), the effect of double-layer polarization can be simulated by considering

$$
n_{j}=n_{j 0} \exp \left[\frac{-z_{j} e}{k T}\left(\phi_{1}+\phi_{2}+g_{j}\right)\right], \quad j=1,2,
$$

where $g_{j}$ denotes a perturbation term which accounts for the effect of fluid flow on the concentration of ions. If $\phi_{2}$ and $g_{j}$ are small compared to both $k T / e$ and $\phi_{1}$, Eq. [7] can be approximated by

$n_{j}=n_{j 0} \exp \left(-\frac{z_{j} e \zeta_{a}}{k T} \phi_{1}^{*}\right)\left[1-\frac{z_{j} e \zeta_{a}}{k T}\left(\phi_{1}^{*}+g_{j}^{*}\right)\right], \quad j=1,2$

where $\phi_{j}^{*}=\phi_{j} / \zeta_{a}, g_{j}^{*}=g_{j} / \zeta_{a}, j=1,2, \zeta_{a}$ being the zeta potential. $\phi_{1}^{*}$ represents the scaled electric potential at equilibrium. As pointed out by O'Brien and White (13), that $\phi_{2}$ and $g_{j}$ are small does not imply that their effects are insignificant.

For an easier mathematical treatment, scaled properties are used in the following analyses where the radius of the representative cylinder $a$ is chosen as the characteristic length and $U_{\mathrm{E}}\left(=\varepsilon \zeta_{a}^{2} / \mu a\right)$ is chosen as the characteristic velocity. The latter is the electrophoretic velocity of an isolated cylinder evaluated by Smoluchowski's theory (14).

Adopting the approach of Lee et al. (7), Eq. [4] is transformed by taking curl to yield the governing equation for the corresponding stream function $\Psi$. The $r$ and $\theta$ components of $\vec{v}, v_{r}$ and $v_{\theta}$, can be evaluated from $\Psi$ by $v_{r}=-(\partial \Psi / \partial \theta) / r$ and $v_{\theta}=\partial \Psi / \partial r$, respectively. For convenience, a scaled stream potential $\Psi^{*}=\Psi / a U_{\mathrm{E}}$ is defined. The symmetric nature of 
the problem suggests that $\phi_{2}^{*}, g_{1}^{*}, g_{2}^{*}$, and $\Psi^{*}$ can be expressed respectively by $\phi_{2}^{*}=\Phi_{2}(r) \cos \theta, g_{1}^{*}=G_{1}(r) \cos \theta$, $g_{2}^{*}=G_{2}(r) \cos \theta$, and $\Psi^{*}=\Omega(r) \sin \theta$. It can be shown that the variation of $\phi_{1}^{*}$ is described by (7)

$$
L \phi_{1}^{*}=-\frac{(\kappa a)^{2}}{(1+\alpha) \phi_{r}}\left[\exp \left(-\phi_{r} \phi_{1}^{*}\right)-\exp \left(\alpha \phi_{r} \phi_{1}^{*}\right)\right]
$$

where the scaled surface potential $\phi_{r}$, the linear operator $L$, and the Debye-Huckel parameter $\kappa$ are defined, respectively, by

$$
\begin{gathered}
\phi_{r}=\zeta_{a} z_{1} e / k T \\
L=\frac{d^{2}}{d r^{* 2}}+\frac{1}{r^{*}} \frac{d}{d r^{*}}-\frac{1}{r^{* 2}}
\end{gathered}
$$

and

$$
\kappa^{2}=\frac{e \sum n_{i 0} z_{i}}{\varepsilon k T}=\frac{(1+\alpha) e^{2} n_{10} z_{1}^{2}}{\varepsilon k T} .
$$

We assume that the unit cell as a whole is electrically neutral and that there is no net current flow between adjacent cells. Therefore the boundary conditions associated with Eq. [9] are

$$
\begin{aligned}
\phi_{1}^{*} & =1 \quad \text { at } r^{*}=1 \\
\frac{d \phi_{1}^{*}}{d r^{*}} & =0 \quad \text { at } r^{*}=b / a .
\end{aligned}
$$

We need to solve Eq. [8] along with the following equations (7):

$$
\begin{gathered}
{\left[L-\frac{(\kappa a)^{2}}{1+\alpha}\left[\exp \left(-\phi_{r} \phi_{1}^{*}\right)+\alpha \exp \left(\alpha \phi_{r} \phi_{1}^{*}\right)\right]\right] \Phi_{2}} \\
=\frac{(\kappa a)^{2}}{1+\alpha}\left[G_{1} \exp \left(-\phi_{r} \phi_{1}^{*}\right)+\alpha G_{2} \exp \left(\alpha \phi_{r} \phi_{1}^{*}\right)\right] \\
L G_{1}-\phi_{r} \frac{d \phi_{1}^{*}}{d r^{*}} \frac{d G_{1}}{d r^{*}}=\operatorname{Pe}_{1} v_{r}^{*} \frac{d \phi_{1}^{*}}{d r^{*}} \\
L G_{2}+\alpha \phi_{r} \frac{d \phi_{1}^{*}}{d r^{*}} \frac{d G_{2}}{d r^{*}}=\operatorname{Pe}_{2} v_{r}^{*} \frac{d \phi_{1}^{*}}{d r^{*}} \\
\nabla^{4} \Omega=-\frac{1}{r^{*}} \frac{(\kappa a)}{1+\alpha}\left[\left(n_{1}^{*} G_{1}+\alpha n_{2}^{*} G_{2}\right) \frac{d \phi_{1}^{*}}{d r^{*}}\right] .
\end{gathered}
$$

In these expressions $\operatorname{Pe}_{j}=\varepsilon\left(z_{1} e / k T\right)^{2} / \mu D_{j}, j=1,2$, is the electric Peclet number of ion species $j, n_{1}^{*}=\exp \left(-\phi_{r} \phi_{1}^{*}\right)$, $n_{2}^{*}=\exp \left(\alpha \phi_{r} \phi_{1}^{*}\right)$, and the operator $\nabla^{4}$ is defined by

$$
\nabla^{4}=\left(\nabla^{2}\right)^{2}=\left(\frac{d^{2}}{d r^{* 2}}+\frac{1}{r^{*}} \frac{d}{d r^{*}}-\frac{1}{r^{* 2}}\right)^{2}
$$

We assume the following: (a) the representative cylinder is nonconducting, (b) ion species achieve the equilibrium value at the virtual surface $(r=b)$, (c) the fluid is moving at constant velocity $U$, and (d) the virtual surface is steady and satisfies Kuwabara's model of zero vorticity. Therefore the boundary conditions associated with Eqs. [10]-[13] are

$$
\begin{aligned}
& \frac{d \Phi_{2}}{d r^{*}}=0 \quad \text { at } r^{*}=1 \\
& \frac{d \Phi_{2}}{d r^{*}}=-E_{\mathrm{x}}^{*} \quad \text { at } r^{*}=b / a \\
& \frac{d G_{j}}{d r^{*}}=0 \quad \text { at } r^{*}=1, j=1,2 \\
& G_{j}=-\Phi_{2} \quad \text { at } r^{*}=b / a, j=1,2 \\
& \Omega=0 \quad \text { and } \quad \frac{d \Omega}{d r^{*}}=0 \quad \text { at } r^{*}=1 \\
& L \Omega=0 \quad \text { and } \quad \Omega=U r^{*} \quad \text { at } r^{*}=b / a \text {. }
\end{aligned}
$$

In these expressions $L=\nabla^{2}, E_{x}^{*}=E_{x} a / \zeta_{a}$, and $U^{*}=U / U_{\mathrm{E}}$, $E_{\mathrm{x}}$ and $U$ being, respectively, the x component of the electric field and the bulk velocity of liquid. The choice of zero vorticity rather than zero shear stress at the cell boundary is based on the consideration that the former leads to Smoluchowski's result for an isolated charged fiber. The choice of the boundary condition expressed in Eq. [13c] is based on the assumption of Levine and Neale (1); that is, the local electric field is parallel to the external applied field at $r=b$. We have $-\nabla \phi_{2}=\vec{E}_{\mathrm{x}}$ or $-\nabla^{*} \phi_{2}^{*}=\vec{E}_{\mathrm{x}}$. Therefore, $\left(\partial \phi_{2}^{*} / \partial r\right)_{r^{*}=b / a}=$ $-E_{\mathrm{x}}^{*} \cos \theta$. Since $\phi_{2}^{*}=\Phi_{2} \cos \theta$ at $r^{*}=b / a$, Eq. [13c] is justified.

At steady state the sum of the $\mathrm{x}$ components of all the forces, including the electrical force $F_{\mathrm{Ex}}$ and the viscous force $F_{\mathrm{Dx}}$, acting on a unit length of the representative cylinder vanishes. The former can be calculated by $F_{\mathrm{Ex}}=\iint_{S} \sigma(-\nabla \phi) d A$, and the latter can be evaluated by the method used in Kozak and Davis (8). It can be shown that

$$
\begin{aligned}
F_{\mathrm{Ex}}= & \frac{2 \varepsilon \zeta_{a}^{2}}{a} \int_{0}^{\pi}\left(\frac{\partial \phi^{*}}{\partial r^{*}}\right)_{S}\left(\frac{\partial \phi^{*}}{\partial r^{*}} \cos \theta-\frac{1}{r^{*}} \frac{\partial \phi^{*}}{\partial \theta} \sin \theta\right)_{S} r^{*} d \theta \\
= & \frac{\pi \varepsilon \zeta_{a}^{2}}{a}\left(\frac{d \phi_{1}^{*}}{d r^{*}}\right)_{r^{*}=1}\left(\Phi_{2}^{*}\right)_{r^{*}=1} \\
F_{\mathrm{Dx}}= & 2 \int_{0}^{\pi}\left[-p \cos \theta+2 \mu \frac{\partial V_{r}}{\partial r} \cos \theta+\tau_{r \theta} \sin \theta\right]_{r=a} r d \theta \\
= & \frac{\pi \varepsilon \zeta_{a}^{2}}{a}\left[r^{* 2} \frac{d^{3} \Omega}{d r^{* 3}}-3 \frac{d \Omega}{d r^{*}}+\frac{3}{r^{*}} \Omega\right]_{r^{*}=1} \\
& +\left[\frac{\pi \varepsilon \zeta_{a}^{2}}{a} \frac{(\kappa a)^{2}}{(1+\alpha)} r^{*}\left(n_{1}^{*}-n_{2}^{*}\right) \Phi_{2}\right]_{r^{*}=1} .
\end{aligned}
$$

According to O'Brien and White (13), the present linear problem can be decomposed into two subproblems. In the first problem the fluid moves at a constant velocity $U$ in the absence of the applied electric field, and in the second problem the fluid is fixed in the applied electric field. The force required to move the fluid in the first problem, $f_{1}$, can be expressed as $f_{1}=\delta U^{*}$, and that exerts on the fluid in the second problem, $f_{2}$, as $f_{2}=\beta E_{\mathrm{x}}^{*}$. 
That the net force vanishes implies that $U_{0}^{*}=U^{*} / E_{x}^{*}=-\beta / \delta$, $U_{0}^{*}$ being the scaled electroosmotic velocity.

\section{RESULTS AND DISCUSSIONS}

Figure 2 shows the variation of the scaled electroosmotic velocity, $U_{0}^{*}$, as a function of $\kappa a$ at various scaled surface potential $\phi_{r}$ for the case $H=0.75$. Double-layer polarization is neglected. The results of Ohshima (9) are also shown for comparison. As can be seen from Fig. 2, for fixed $\phi_{r}, U_{0}^{*}$ increases with $\kappa a$. This is because for a fixed cylinder radius, the smaller the $\kappa a$, the thicker the double layer, and, therefore, the more the electrical interaction between adjacent cylinders. For a fixed $\kappa a, U_{0}^{*}$ increases with $\phi_{r}$ as expected. Figure 2 also suggests that if the effect of double-layer polarization is neglected, that is, the result of Ohshima, using the linearized Poisson-Boltzmann equation will underestimate the electroosmotic velocity. The deviation becomes small, however, if $\kappa a$ is either very large or very small. If $\kappa a$ is very large, the result of Ohshima can be recovered from the present study; that is, if the double layer is thin, the effect of the double layer is insignificant, as expected. Also, although not shown in Fig. 2, $U_{0}^{*} \rightarrow 1$ as $\kappa a \rightarrow \infty$.

Figures 3 and 4 show the variation of scaled electroosmotic velocity $U_{0}^{*}$ as a function of $\kappa a$ at various scaled surface potential $\phi_{r}$ for two levels of $H\left(=1-(a / b)^{2}\right)$ for the case where doublelayer polarization is considered. Also shown in these figures are the results based on Ohshima (9). Figures 3 and 4 reveal that if $\kappa a$ is either very small or very large, the result of Ohshima can be recovered by the present analysis. For an intermediate $\kappa a$, depending upon the values of $\kappa a, H$, and $\phi_{r}$, the result of Ohshima may either underestimate or overestimate $U_{0}^{*}$. The deviation of the result of Ohshima from the present result is

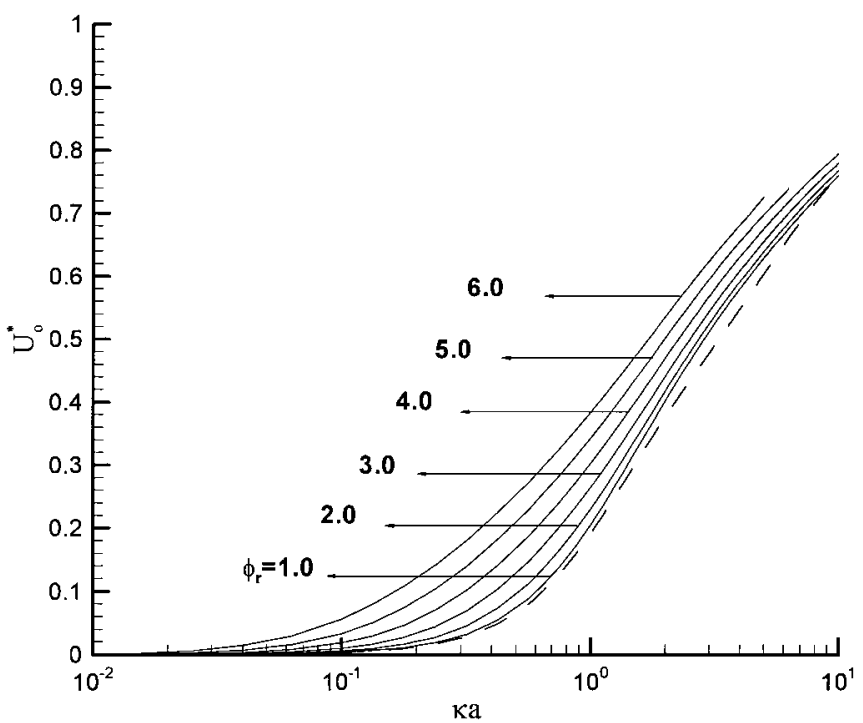

FIG. 2. Variation of scaled electroosmotic velocity $U_{0}^{*}$ as a function of $\kappa a$ at various scaled surface potential $\phi_{r}$ for the case $H=0.75$. Double-layer polarization is neglected. Key: $a=1, b=2, \alpha=1$, and $\mathrm{Pe}_{1}=\mathrm{Pe}_{2}=0.1$. Dashed line, result of Ohshima (9); solid lines, present study.

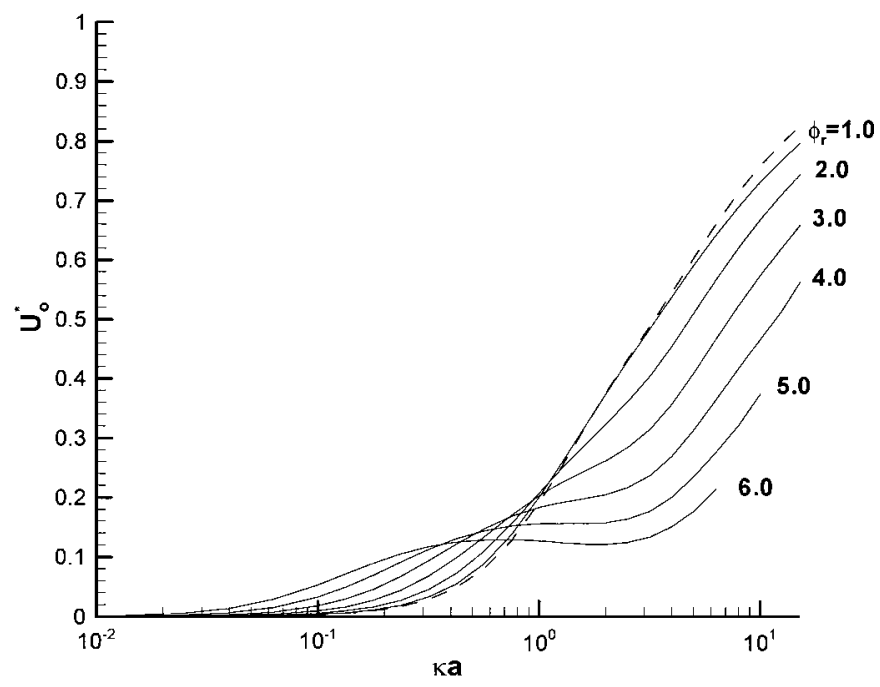

FIG. 3. Variation of scaled electroosmotic velocity $U_{0}^{*}$ as a function of $\kappa a$ at various scaled surface potential $\phi_{r}$ for the case $H=0.75$. Double-layer polarization is considered. Key: same as that in Fig. 2.

the net result of two factors. As discussed in Fig. 2, using the linearized Poisson-Boltzmann equation will underestimate $U_{0}^{*}$. On the other hand, according to Kozak and Davis (8), the electric field induced by the representative cylinder has the effect of lessening the applied electric field. For fixed $\phi_{r}$, the thinner the double layer, the greater the gradient of electrical potential, and the stronger the induced electric field. Therefore, if $\kappa a$ is large, the result of Ohshima will overestimate $U_{0}^{*}$, and the reverse is true if $\kappa a$ is small. Note that if $\phi_{r}$ is sufficiently high, there may exist a local maximum and a local minimum. A comparison between Figs. 3 and 4 shows that for a fixed $\kappa a, U_{0}^{*}$ increases with the increase in $H$. This is because the larger the $H$ the higher the porosity of the fibrous medium and, therefore, the

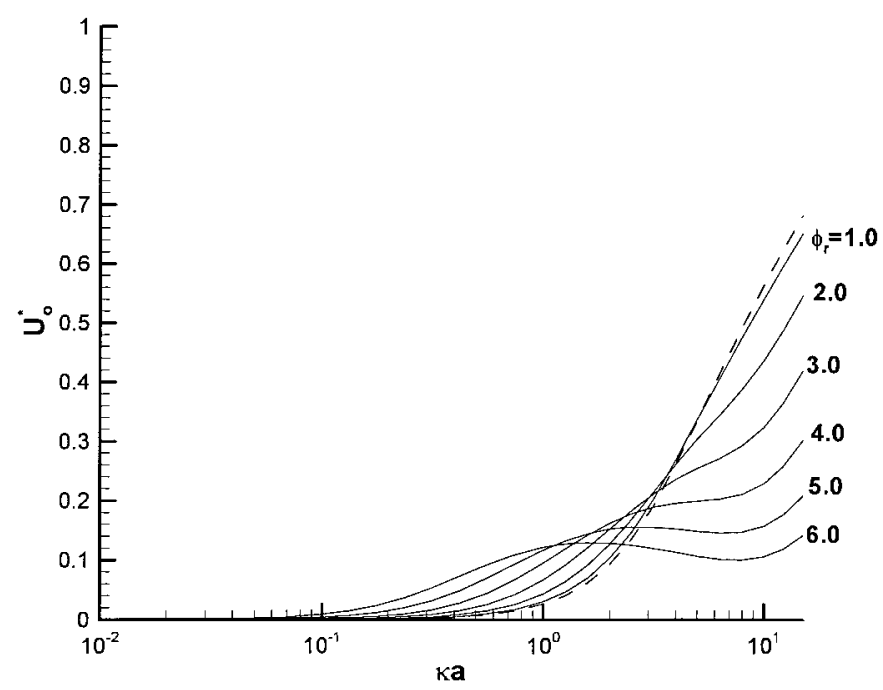

FIG . 4. Variation of scaled electroosmotic velocity $U_{0}^{*}$ as a function of $\kappa a$ at various scaled surface potential $\phi_{r}$ for the case $H=0.408$. Double-layer polarization is considered. Key: same as that in Fig. 2, except $b=1.3$. 


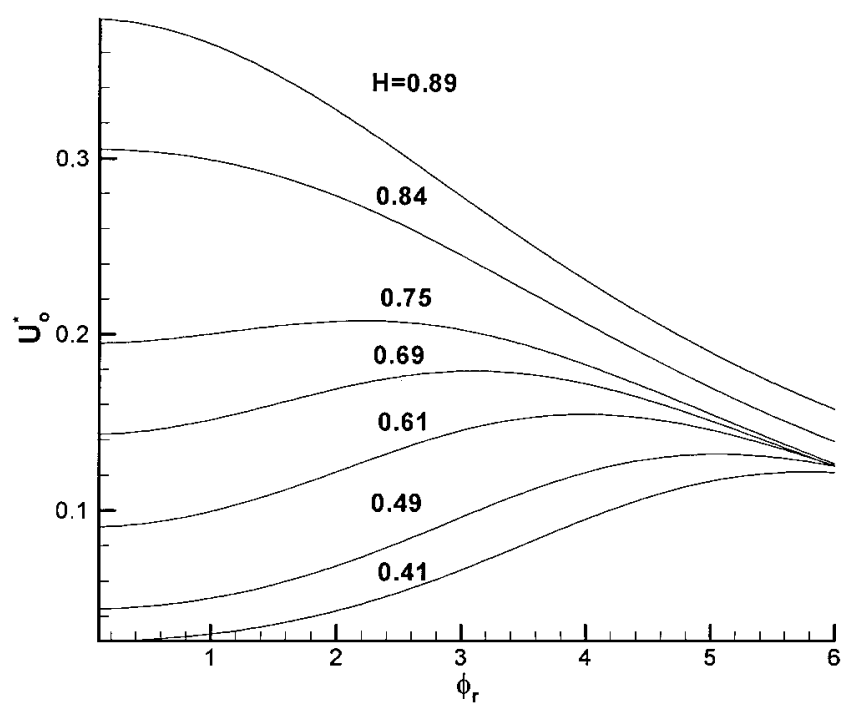

FIG . 5. Variation of scaled electroosmotic velocity $U_{0}^{*}$ as a function of $\phi_{r}$ at various $H$ for the case $\kappa a=1, \alpha=1$, and $\mathrm{Pe}_{1}=\mathrm{Pe}_{2}=0.1$.

smaller the interference between adjacent fibers and, therefore, the greater the $U_{0}^{*}$. Note that the larger the $H$ the lower the level of $\kappa a$ required for the local extremals to occur.

Figure 5 illustrates the variation of scaled electroosmotic velocity $U_{0}^{*}$ as a function of $\phi_{r}$ for fixed $\kappa a$. This figure reveals that if $H$ is sufficiently small, $U_{0}^{*}$ has a local maximum as $\phi_{r}$ varies. We find that the value of $H$ at which the local maximum exists decreases with $\kappa a$.

The variations of scaled electroosmotic velocity $U_{0}^{*}$ as a function of $H$ at two different $\kappa a$ and two levels of $\phi_{r}$ are presented in Figs. 6 and 7. Also illustrated in these figures are the corresponding results based on the analysis of Ohshima (9). Figure 6 shows that for a fixed $\kappa a, U_{0}^{*}$ increases with $H$, and, for a fixed $H, U_{0}^{*}$ increases with $\kappa a$. The difference between the present

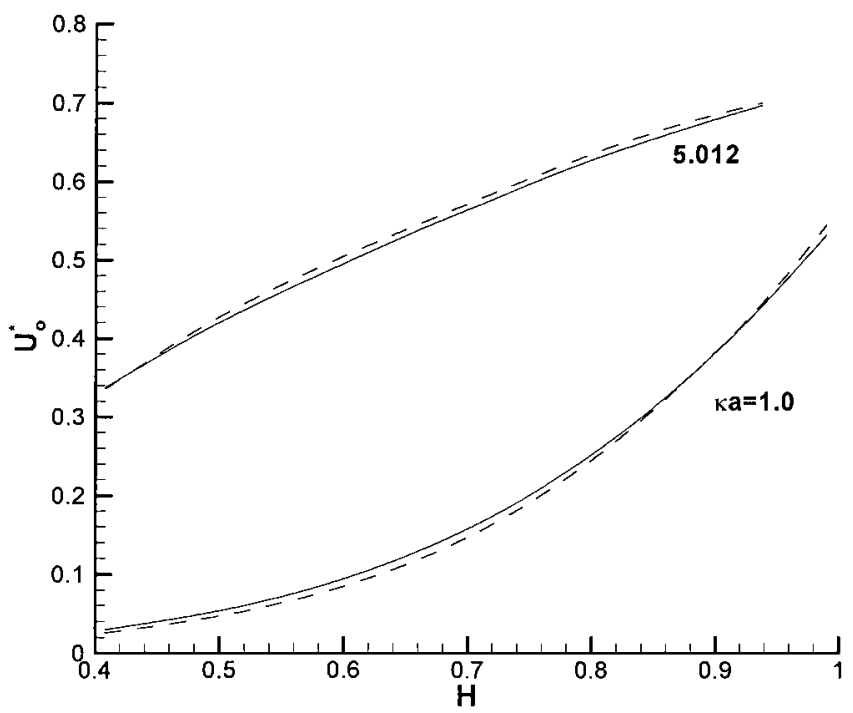

FIG. 6. Variation of scaled electroosmotic velocity $U_{0}^{*}$ as a function of $H$ at two different $\kappa a$ for the case $\phi_{r}=1$. Key: same as that in Fig. 2.

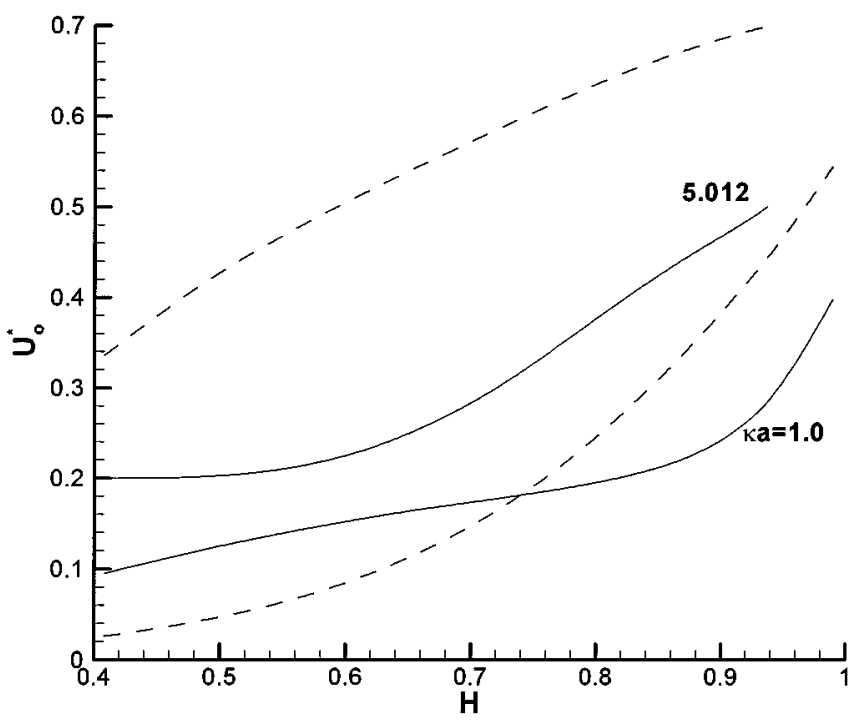

FIG. 7. Variation of scaled electroosmotic velocity $U_{0}^{*}$ as a function of $H$ at two different $\kappa a$ for the case $\phi_{r}=4$. Key: same as that in Fig. 2.

result and that of Ohshima is inappreciable at $\phi_{r}=1$. The difference becomes appreciable, however, for a higher level of $\phi_{r}$, as can be seen in Fig. 7. This figure also reveals that if $\kappa a$ is small, the effect of surface potential on the electroosmotic velocity is more significant than that of double-layer polarization. Also, the result of Ohshima will underestimate $U_{0}^{*}$. On the other hand, if $\kappa a$ is large, the reverse is true. For a high $H$ and a medium $\kappa a$, the effect of double-layer polarization on $U_{0}^{*}$ is more significant than that of surface potential.

The variations of scaled electroosmotic velocity $U_{0}^{*}$ as a function of $\kappa a$ for various $H$ for two $\phi_{r}$ are shown in Figs. 8 and 9. The corresponding results based on the model of Ohshima (9) are also presented in the former for comparison. Again, if $\phi_{r}$

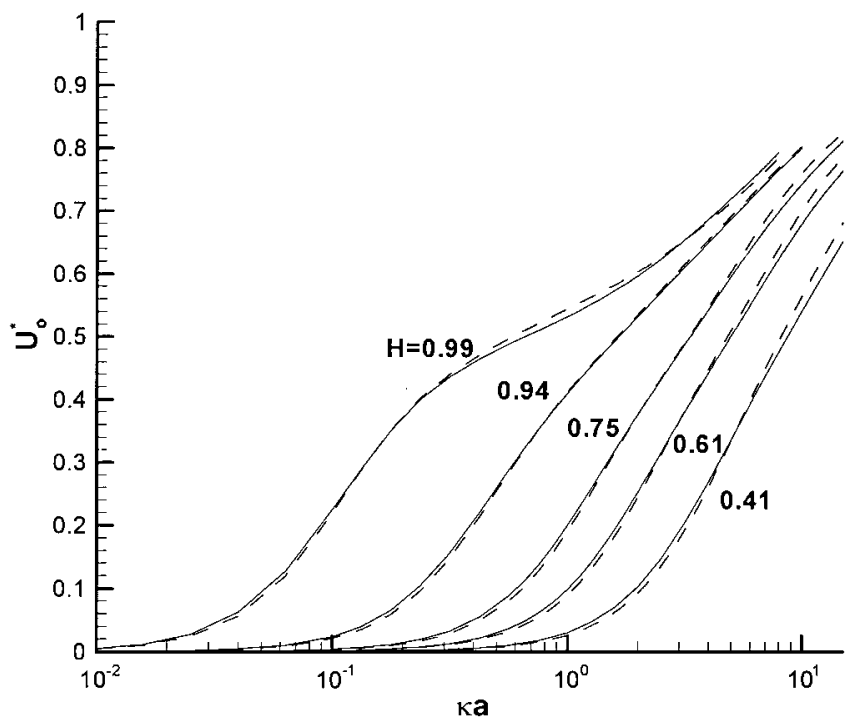

FIG. 8. Variation of scaled electroosmotic velocity $U_{0}^{*}$ as a function of $\kappa a$ for various $H$ for the case $\phi_{r}=1$. Key: $a=1, b=2, \mathrm{Pe}_{1}=\mathrm{Pe}_{2}=0.1$, and $\alpha=1$. Dashed lines are the results of Ohshima (9). 


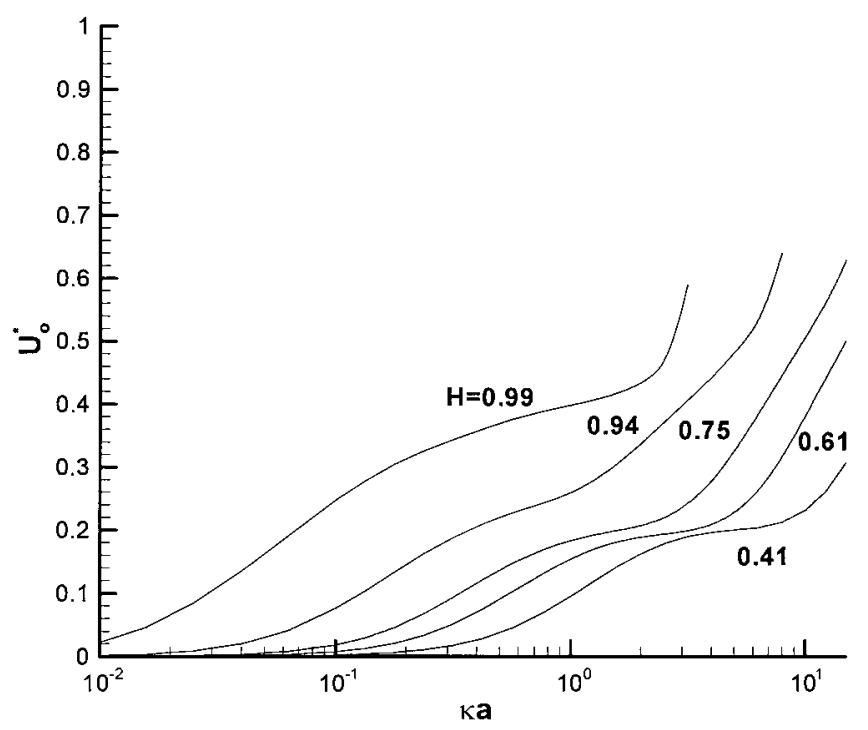

FIG. 9. Variation of scaled electroosmotic velocity $U_{0}^{*}$ as a function of $\kappa a$ for various $H$ for the case $\phi_{r}=4$. Key: same as that in Fig. 8 .

is small, the difference between the present model and that of Ohshima is insignificant, as illustrated in Fig. 8. On the other hand, if $\phi_{r}$ is sufficiently high, the variation of $U_{0}^{*}$ as a function of $\kappa a$ may have local maximum and minimum, as can be seen in Fig. 9.

The major contribution of the present work can be summarized as follows. Levine and Neale (1-3) investigated both the electrophoretic and electroosmotic behaviors of a system containing multiple spheres. The result of Smoluchowski (14) was extended to the case of an arbitrary thickness of double layer, but was limited to a low level of electrical potential. Kozak and Davis (8) used the linearized Poisson-Boltzmann equation and neglected double-layer polarization and overlapping, but the result obtained in the present analysis is applicable to an arbitrarily thick double layer. The integral representation for electroosmotic velocity in Kozak and Davis (8) was simplified by Ohshima (9), and an approximate analytical expression was derived. In the present study, the electrical potential can assume an arbitrary level, and the effects of double-layer polarization and the overlapping of adjacent double layers are taken into account.

\section{ACK NOWLEDGMENT}

This work is supported by the National Science Council of the Republic of China.

\section{REFERENCES}

1. Levine, S., and Neale, G. H., J. Colloid Interface Sci. 47, 520 (1974).

2. Levine, S., and Neale, G. H., J. Colloid Interface Sci. 49, 330 (1974).

3. Levine, S., and Neale, G. H., J. Colloid Interface Sci. 57, 424 (1976).

4. Kozak, M. W., and Davis, E. J., J. Colloid Interface Sci. 127, 497 (1989).

5. Kozak, M. W., and Davis, E. J., J. Colloid Interface Sci. 129, 166 (1989).

6. Ohshima, H., J. Colloid Interface Sci. 188, 481 (1996).

7. Lee, E., Chu, J. W., and Hsu, J. P., J. Colloid Interface Sci., 209, 240 (1999).

8. Kozak, M. W., and Davis, E. J., J. Colloid Interface Sci. 112, 403 (1986).

9. Ohshima, H., J. Colloid Interface Sci. 210, 397 (1999).

10. Lee, E., Chu, J. W., and Hsu, J. P., J. Colloid Interface Sci., 205, 65 (1998).

11. Lee, E., Chu, J. W., and Hsu, J. P., J. Colloid Interface Sci., 196, 316 (1997).

12. Kuwabara, S., J. Phys. Soc. Jpn. 14, 527 (1959).

13. O'Brien, R. W., and White, L. R., J. Chem. Soc. Faraday II. 74, 1607 (1978).

14. Smoluchowski, M., Z. Phys. Chem. 93, 129 (1918). 